\title{
Synthetic Diversity and Catalytic Mechanism of Peptide Dendrimers
}

\author{
Estelle Delort\$, Tamis Darbre, and Jean-Louis Reymond ${ }^{\star}$ \\ $\S$ Mettler Toledo Award Winner (Oral Presentation)
}

\begin{abstract}
Peptide dendrimers composed of alternating sequences of natural amino acids and branching diamino acids are investigated as synthetic enzyme models. The dendrimers can be prepared by solid-phase peptide synthesis and are obtained pure in yields of 5-35\%. Peptide dendrimers with surface histidine residues catalyze ester hydrolysis reaction with enzyme-like kinetics, including substrate binding $\left(K_{\mathrm{M}}\right)$, catalytic turnover $\left(k_{\text {cat }}\right)$, and rate acceleration $k_{\text {cat }} / k_{\text {uncat }}=1000-20$ '000. Mechanistic investigation by substrate variation, $\mathrm{pH}$-profile, and isothermal titration calorimetry show that the catalytic effect is caused by positive interaction between the histidine side-chains and creation of a hydrophobic microenvironment for substrate binding.
\end{abstract}

Keywords: Dendrimer · Enzyme model $\cdot$ Ester hydrolysis $\cdot$ Peptide $\cdot$ Solid-phase synthesis

\section{Introduction}

Dendrimers are branched molecules composed of three components: the core, the branches, and the periphery [1]. Dendrimers are under investigation for a wide range of applications in homogeneous catalysis, drug and gene delivery, and nanotechnology [2]. Dendritic peptides such as polylysine have been known for many years and are used for multiple antigenic displays [3]. We have shown recently that peptide dendrimers can be prepared by divergent synthesis on solid support, which enables structural diversity at the core, periphery, and within the branches. Our contribution was first to establish that dendrimer synthesis with various sequences was possible on solid support with efficiencies comparable to that of linear peptides, and allowed the installation of structural diversity throughout the dendrimer structure.

${ }^{\star}$ Correspondence: Prof. J.-L. Reymond University of Bern

Department of Chemistry and Biochemistry

Freiestrasse 3

$\mathrm{CH}-3012$ Bern

Tel: : +41316314325

Fax: +41316318057

E-mail: jean-louis.reymond@ioc.unibe.ch

http://www.dcb.unibe.ch/groups/reymond/
Our first series of investigations with peptide dendrimers was based on a dimerization strategy involving disulfide bond formation between two second-generation dendrimers containing three variable amino acid positions at the core ( $\mathrm{A}^{1}$, one copy), first generation ( $\mathrm{A}^{2}$, two copies), and second generation $\left(\mathrm{A}^{3}\right.$, four copies) (Scheme 1 design A) [4] . Accessing structural diversity by synthesis enabled us to survey small libraries of dendrimers for possible proteinlike functions. Indeed the dendritic architecture enforces a globular shape by design, which might lead to productive interactions between amino acids as in proteins. Peptide dendrimers bearing histidine side-chains were found to catalytically hydrolyze simple fluorogenic esters (Scheme 2). The dendrimers showed typical enzyme-like kinetic parameters in water with substrate recognition $\left(K_{\mathrm{M}}\right)$ and turnover $\left(k_{\text {cat }}\right)$. However catalysis was limited to dendrimers displaying the critical histidine side-chain at the surface of the dendrimers.

The original disulfide-bridged design allowed six individually variable positions. However the architecture and synthetic route chosen was limited in terms of combinatorial diversity because each dendrimer had to be assembled separately in solution and purified. To access structural diversity more efficiently we have turned to a slightly different design based on branches incorporating two amino acids instead of only one. This modification allows the synthesis of higher generation dendrimers with a much greater structural diversity, and opens the route to the discovery of truly efficient peptide dendrimer catalysts. Herein we discuss the synthesis and mechanism of esterolytic dendrimers discovered in this new dendrimer family.

\section{Synthesis of Peptide Dendrimers}

The architecture chosen incorporates two variable amino acids in each branch, with the first branch representing the dendritic core, and the last branch representing the dendritic surface (Scheme 1, design B) [5]. Peptide dendrimers with this type of branching sequence can be obtained in excellent yields by Fmoc-type solid-phase peptide synthesis on Tentagel Rink amide resin using commercially available amino acid and diamino acid building blocks. Cleavage from the resin and purification by preparative reverse-phase HPLC gives pure dendrimers with yields ranging between $5 \%$ and $35 \%$. Considering that a third-generation dendrimer with this architecture contains thirty-seven amino acids, the synthesis yields must be considered excellent if not superior to those achievable for linear peptides of similar size. A typical synthetic scheme is shown in Scheme 3. Structural investigations of peptide dendrimers by nuclear magnetic resonance are currently underway. We are also establishing an automated protocol for parallel synthesis of peptide dendrimers using a peptide synthesizer in collaboration with Chemspeed AG in Augst, Switzerland. 


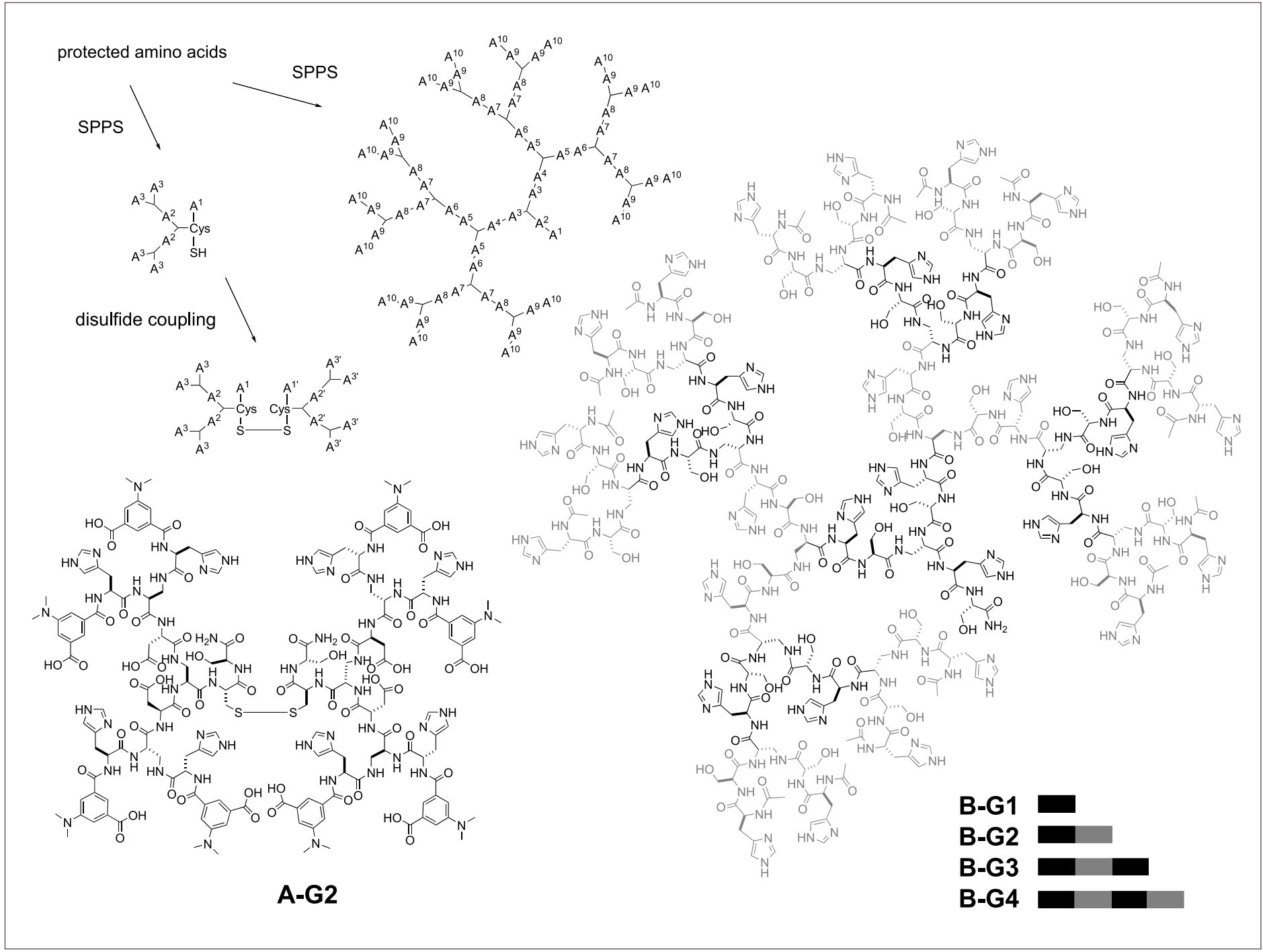

Scheme 1. Two different peptide dendrimer architectures. The branching points consist of (S)-2,3-diaminopropanoic acid. In design $\mathbf{A}$, the $\mathbf{N}$ terminus at position $\mathrm{A}^{3}$ is acylated with the 3 -dimethylaminoisophthaloyl group, introduced as the mono-tert-butyl ester. In design $\mathbf{B}$, the $\mathbf{N}$ terminus at position $\mathrm{A}^{10}$ is acetylated with acetic anhydride. SPPS = solid-phase peptide synthesis. Gn refers as the number $\mathbf{n}$ of dendritic generations.<smiles>CC(C)C(=O)Oc1ccc2ccc[n+](C)c2c1</smiles>
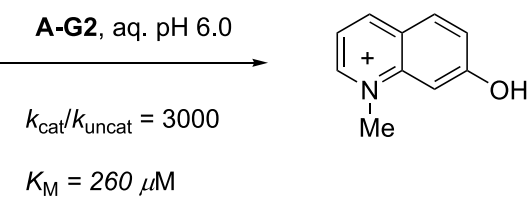

Scheme 2. Peptide dendrimer A-G2 - catalyzed N-methylquinolinium ester hydrolysis with Michaelis-Menten parameters. Conditions: 0.04-0.7 mM ester substrate 1, $5 \mu \mathrm{M}$ A-G2, $20 \mathrm{mM}$ aq. BisTris pH 6.0, $25^{\circ} \mathrm{C}$. The kinetic constants given are derived from the linear double reciprocal plots of 1/Vnet versus 1/[S].

\section{Dendritic Structural Diversity}

The diversity of protein shapes and functions is made possible by the structural diversity resulting from various combinations of amino acids along the peptide chains. For peptide dendrimers the overall shape should be approximately constant as

imposed by the branching structure. A large diversity is possible by variation of the amino acids in the branches, which can include both proteinogenic and non-proteinogenic amino acids. A quantitative estimation of this diversity is informative as to the potential of the peptide dendrimer approach to supramolecular protein models (Table). For disulfide-bridged second-generation dimers of type A-G2, three different positions can be addressed in the divergent synthesis of each half-dendrimer. This gives six variable positions in the dimer, and a potential diversity of 32 million disulfide-bridged dimers if one uses combinations of 20 different amino acids at each position. The total possible diversity allowed by the structure is however much larger. Indeed if one uses an asymmetric diamino acid branching unit such as 2,3-diaminopropanoic acid, each of the amino acid positions is in principle unique. This results in 14 different variable amino acid positions in the A-G2 dimer. If a synthetic procedure would allow each of these positions to be addressed individual$1 y$, the total diversity is as high as $1.6 \times 10^{18}$ for 20 different amino acids. For instance, we have shown recently that using asymmetrically protected diamino acid branching units indeed allows 12 of these 14 positions to be varied selectively [6]. 


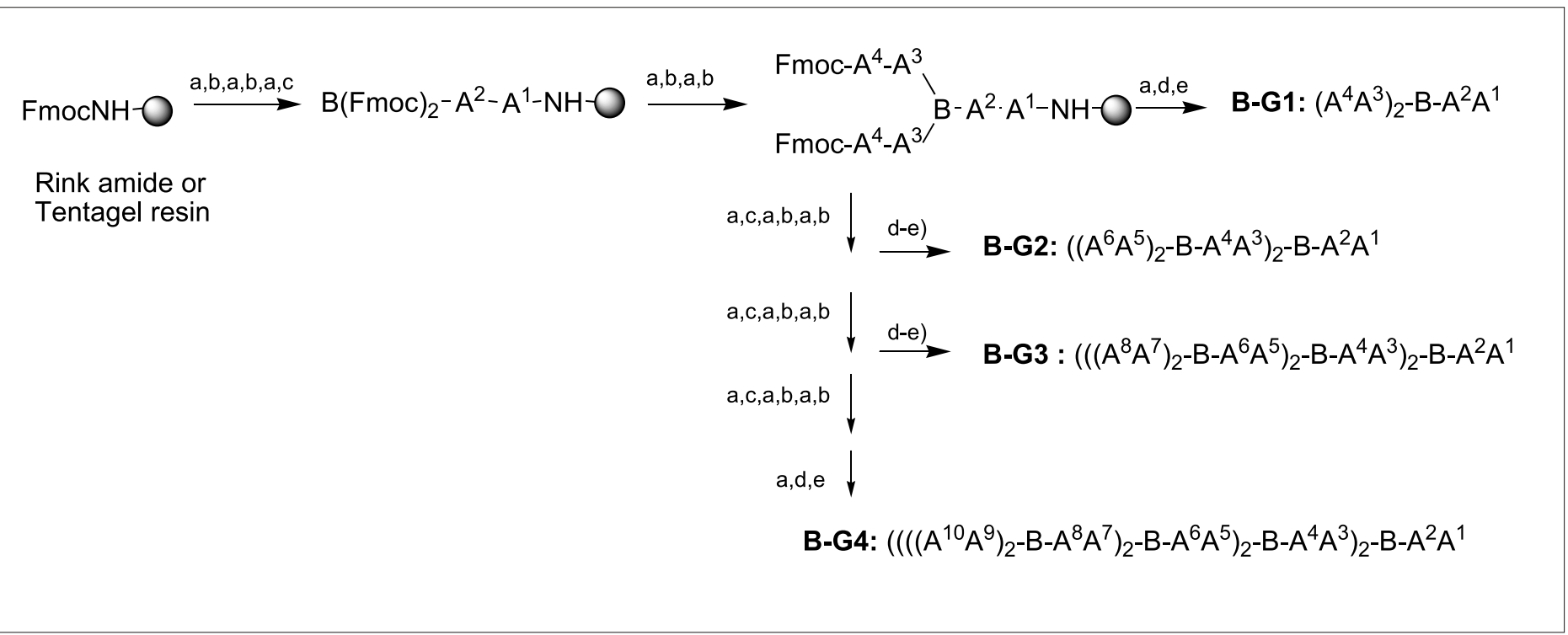

Scheme 3. Synthesis of peptide dendrimers on solid phase. a) DMF/piperidine (4:1), $2 \times 10 \mathrm{~min}$; b) Fmoc-Ai-OH, BOP/DIEA; c) B, BOP/DIEA; d) Ac $\mathrm{O}_{2}$ / DCM (1:1), 1 h; e) TFA/TIS $/ \mathrm{H}_{2} \mathrm{O}(94 / 5 / 1), 4$ h. $\mathrm{A}^{\mathrm{i}}=\alpha$-amino acid, $\mathrm{B}=$ branching diamino acid, Fmoc = 9-fluorenylmethoxycarbonyl, DMF = N,Ndimethylformamide, DIEA = diethylamine, TIS = triisopropylsilane.

Table. Peptide dendrimer diversity from 20 proteinogenic amino acids. The calculation is made for dendrimers with an asymmetric branching diamino acid, e.g. 2,3-diamino propanoic acid, resulting in non-equivalent positions. ${ }^{a}$ Dendrimer structure as in Scheme 1; b Number of variable positions for a layer-by-layer synthesis; cNumber of synthetically accessible dendrimer sequences from 20

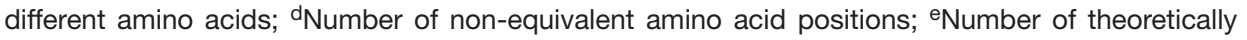
possible dendrimer sequences if each non-equivalent amino acid position would be individually addressable.

\begin{tabular}{lllll}
$\begin{array}{l}\text { peptide } \\
\text { dendrimer }\end{array}$ & $\begin{array}{l}\text { variable } \\
\text { positions }^{\mathrm{b}}\end{array}$ & $\begin{array}{l}\text { synthetic } \\
\text { diversity }\end{array}$ & $\begin{array}{l}\text { amino acid } \\
\text { positions }\end{array}$ & total diversity \\
\hline A-G2 & 6 & $3.2 \times 10^{7}$ & 14 & $1.6 \times 10^{18}$ \\
B-G2 & 6 & $6.4 \times 10^{7}$ & 14 & $1.6 \times 10^{18}$ \\
B-G3 & 8 & $2.6 \times 10^{10}$ & 30 & $1.1 \times 10^{39}$ \\
B-G4 & 10 & $1.0 \times 10^{13}$ & 62 & $4.6 \times 10^{80}$
\end{tabular}

the diad His-Ser at their surface catalyze trisulfonate pyrene ester hydrolysis reactions [5]. Hydrolysis seems to occur by nucleophilic catalysis by the histidine sidechain without participation of serine. To determine the contribution of the dendrimer structure in catalysis, we have investigated a systematic peptide dendrimer series of increasing generation number containing the catalytic sequence His-Ser in all branches (Scheme 1).

Synthesis was carried out on NovaSyn Tentagel resin $(0.25 \mathrm{mmol} / \mathrm{g})$, affording the peptide dendrimers in good yields up to the fourth generation. All dendrimers were catalytically active with multiple turnovers and displayed enzyme-like Michaelis-Menten kinetics (Scheme 4). Remarkably the
Similar arguments can be applied to the structure of type B for the different generation numbers. We have shown recently that combinatorial libraries of dendrimers B-G3 can be prepared by split-and-mix synthesis [7]. The dendrimer library contains over $65^{\prime} 000$ different dendrimers and can screened for binding or catalytic activities directly on solid support. The experiment allows the identification of active dendrimers which can be resynthesized and display the expected properties in solution.

\section{Mechanism of Esterolytic Peptide Dendrimers}

During our investigation of peptide dendrimers as enzyme models, we reported that peptide dendrimers of design $\mathbf{B}$ displaying

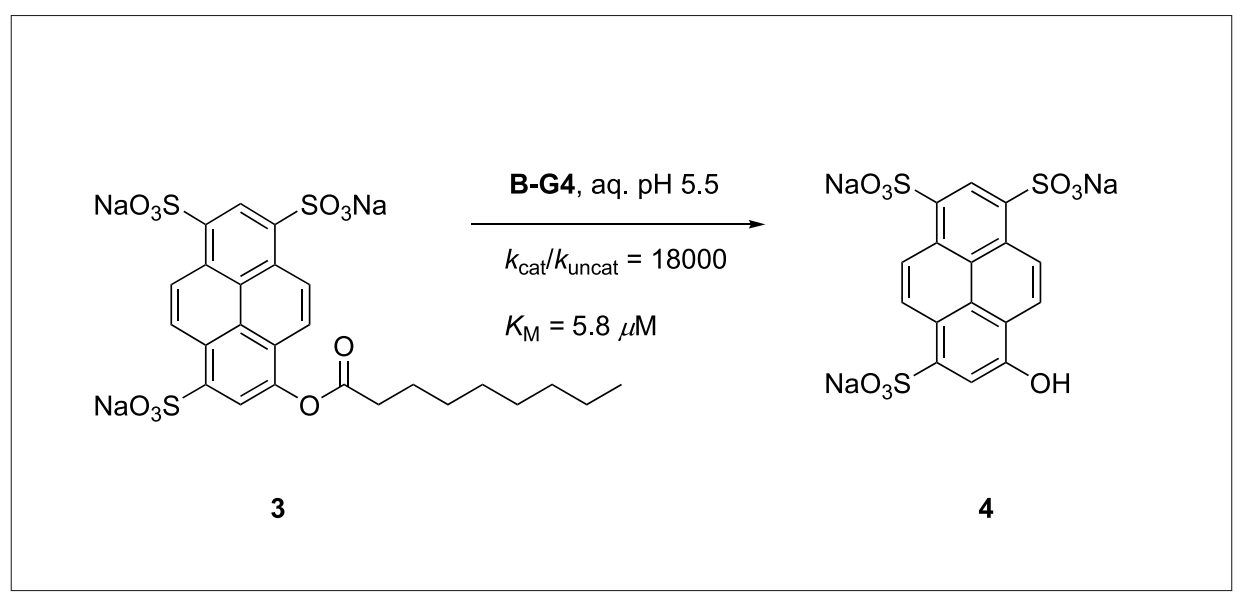

Scheme 4. Peptide dendrimer B-G4 - catalyzed trisulfonate pyrene ester hydrolysis with MichaelisMenten parameters. Conditions: 0.01-1.0 mM ester substrate 3, $1.9 \mu \mathrm{M}$ B-G4, $5 \mathrm{mM}$ aq. citrate pH $5.5,27^{\circ} \mathrm{C}$. The kinetic constants given are derived from the linear double reciprocal plots of $1 / \mathrm{Vnet}$ versus $1 /[\mathrm{S}]$. 
kinetic parameters show that the catalytic efficiency increases with generation despite of the steric crowding of catalytic groups at the periphery, which must occur in higher generation dendrimers.

In the best case of the hydrolysis of the pyrene trisulfonate nonanoyl ester $\mathbf{3}$, the dendrimer B-G1 was 44-fold more efficient per histidine side chain than 4-methyl-imidazole as a reference catalyst, comparable to our previous design A. Additional generations systematically increase the specific rate acceleration over that of 4-methylimidazole per histidine side-chains: from 420fold for B-G2, 1600-fold for B-G3, and up to 4500 -fold for B-G4. This 100 -fold positive dendritic effect between B-G1 and BG4 compares with the very few examples reported so far in the literature [8].

Substrate variations, $\mathrm{pH}$ profile, and isothermal microcalorimetry (ITC) studies show that the strong positive dendritic effect results from cooperative binding and catalysis. The effect of increasing dendrimer size induces catalytically productive interactions such as modulation of the histidine side-chain's $\mathrm{pKa}$, and creation of a hydrophobic microenvironment allowing substrate binding by the acyl chains. Fine-tuning of this dendritic effect toward selective substrate recognition and turnover might enable the preparation of more efficient and selective dendritic catalysts.

\section{Conclusion}

Our experiments show that peptide dendrimers can be prepared in good synthetic yields and purity by solid-phase peptide synthesis. The architecture chosen allows a large structural diversity by variation of the amino acid building blocks, defining a broad family of supramolecules. With this approach it becomes possible to prepare protein-like synthetic structures. The catalytic properties observed are clearly more than the sum of the parts, and indicate effects typical of proteins, such as substrate binding by hydrophobic interactions in water. Such synthetic investigations, using the support of combinatorial methods for discovery, provide a new opportunity to study protein structure and function.

\section{Acknowledgements}

This work was supported by the Swiss National Science Foundation and the University of Bern.

Received: January 6, 2005

[1] a) D.A. Tomalia, Adv. Mater. 1994, 6 , 529; b) G.R. Newkome, C.N. Moorefield, F. Vögtle, Dendritic Molecules: Concepts, Synthesis, Perspectives, VCH, Weinheim, 1996; c) A.W. Bosman, H.M. Janssen, E.W. Meijer, Chem. Rev. 1999, 99, 1665-1688; d) M. Fischer, F. Vögtle, Angew. Chem. 1999, 38, 884-905; e) S.M. Grayson, J.M.J. Fréchet, Chem. Rev. 2001, 101, 3819-3867; f) S. Hecht, J.M.J. Fréchet, Angew. Chem. 2001, 113, 76; Angew. Chem. Int. Ed. 2001, 40, 74; g) H.F. Chow, T.K.-K. Monga, M.F. Nongruma, C.-W. Wan, Tetrahedron 1998, 54, 8543; h) D.A. Tomalia, Aldrichimica Acta 2004, 37, 39-57.

[2] a) Y. Kim, S.C. Zimmerman, Curr. Op. Chem. Biol. 1998, 2, 733-742; b) D.
Astruc, F. Chardac, Chem. Rev. 2001, 101, 2991-3024; c) G.M. Kykes, J. Chem. Technol. Biotechnol. 2001, 76, 903-918; d) J.M.J. Fréchet, J. Polym. Sci. Part A: Polym. Chem. 2003, 41, 3713-3725.

[3] K. Sadler, J.P. Tam, Mol. Biotech. 2002, 90, 195.

[4] a) A. Esposito, E. Delort, D. Lagnoux, F. Djojo, J.-L. Reymond, Angew. Chem. Int. Ed. 2003, 43, 1381-1383; b) D. Lagnoux, E. Delort, C. Douat- Casassus, A. Esposito, J.-L. Reymond, Chem. Eur. J. 2004, 10, 1215-1226; c) C. Douat- Casassus, T. Darbre, J.-L. Reymond, J. Am. Chem. Soc. 2004, 126, 7817-7826.

[5] E. Delort, T. Darbre, J.-L. Reymond, J. Am. Chem. Soc. 2004, 126, 15642-15643.

[6] A. Clouet, T. Darbre, J.-L. Reymond, $A d v$. Synth. Catal. 2004, 346, 1195-1204.

[7] A. Clouet, T. Darbre, J.-L. Reymond, Angew. Chem. Int. Ed. 2004, 43, 46124315.

[8] Positive dendritic effect a) C. Francavilla, M.D. Drake, F.V. Bright, M. Detty, J. Am. Chem. Soc. 2001, 123, 57-67; b) Y. Ribourdouille, G.D. Engel, M. RichardPlouet, L.H. Gade, Chem. Comm. 2003, 1228-1229. 